\title{
Applying Direct Sequence Spread Spectrum to Improve the Throughput of Aloha Protocols
}

\author{
A. I. A. Jabbar \\ College of Engineering - University of Mosul
}

\begin{abstract}
Aloha protocols are characterized by their easy performance and relatively simple hardware requirement as compared with other protocols like CSMA/CA.

The main disadvantages of Aloha protocols are the low throughput and long delays as the offered load increases. In this paper, direct sequence spread spectrum is introduced to increase the throughput of Aloha protocols. This can be achieved by increasing the auto correlation and minimizing the cross correlation and as a consequence decreasing the rate of contention between the transmitted packets.
\end{abstract}

Keywords: Aloha, Slotted Aloha, Direct Sequence, Spread Spectrum.

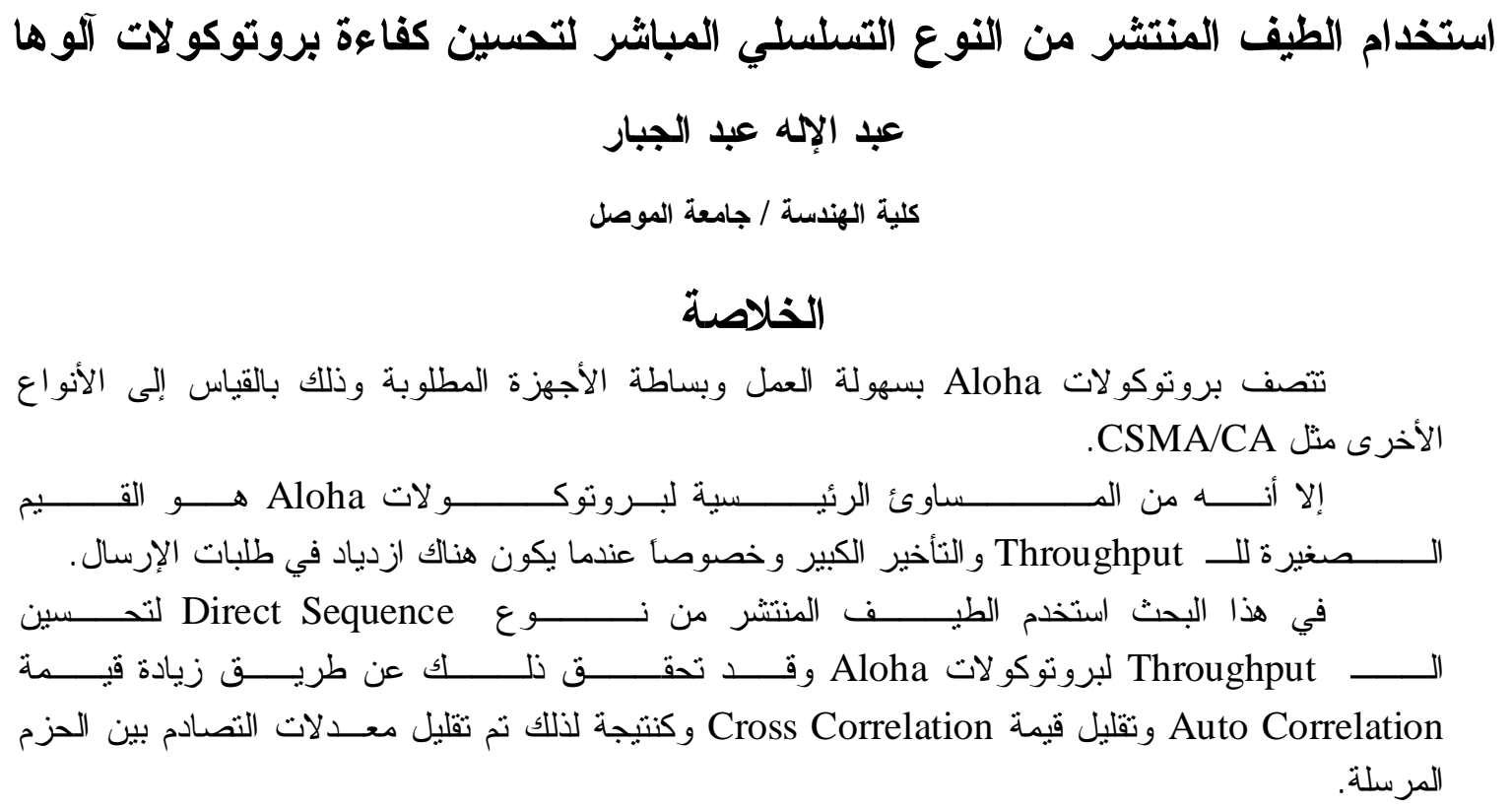




\section{$\begin{array}{llll}\text { Al-Rafidain Engineering } & \text { Vol.17 } & \text { No.5 } & \text { October } 2009\end{array}$}

Introduction:

Conventional pure Aloha is considered as the simplest multiple access protocol being applied practically. According to this protocol, a user with a ready packet will transmit it immediately without regarding the situation of the radio channel (i.e. whether the channel is idle or busy)[1],[2].

It is obvious that there is a possibility of the existence of two users or more transmitting their pockets such that a contention between the packet at the receiving side is resulted, see figure (1).

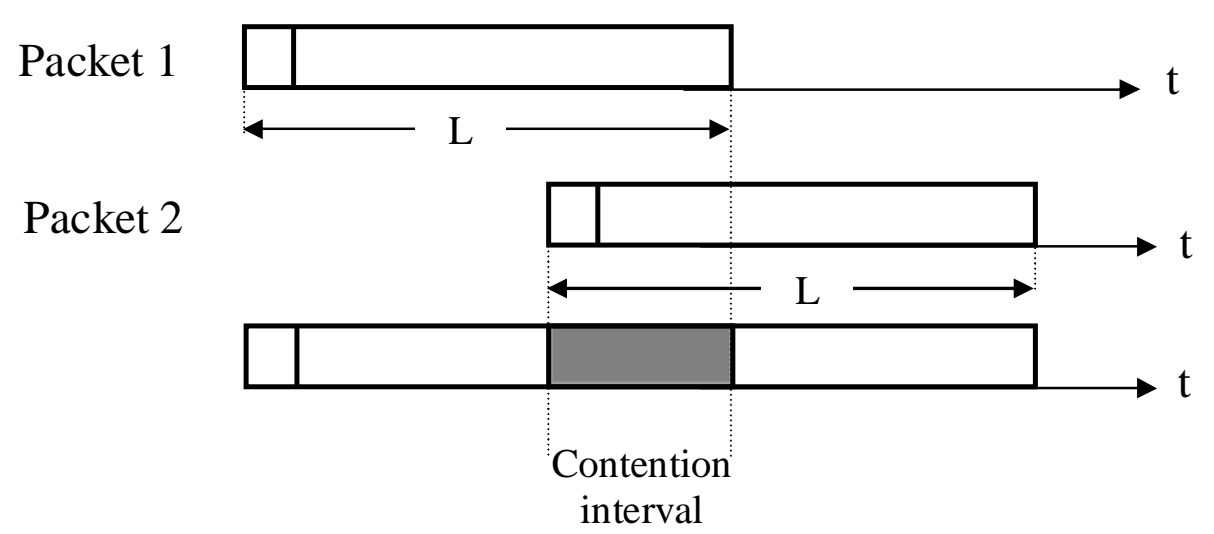

Figure (1) shows two packets subjected to contention problem

In this case, the transmission is considered to be unsuccessful and the transmitting side must retransmit the packets according to a given retransmission algorithm. Practically if any small portion of the packet is collided with other packet, the whole packet must be discarded and it must be retransmitted later. It is clear that the maximum vulnerable period length is equal to (2L), where $\mathrm{L}=$ packet length.

Pure Aloha system can be modeled as a time varying Poisson process, the arrival rate is equal to $(\lambda)$.the starting times of the attempted transmissions can be modeled as a time-varying Poisson process with a rate equal to $\mathrm{G}(\mathrm{n})$. which is given by:

$\mathrm{G}(\mathrm{n})=\lambda+\mathrm{nx}$, when $\mathrm{n}$ is the backlog at a given time.

The probability of successful transmission is related to the fact that there is only one packet being transmitted during the interval (2L). From the probability density function of the Poisson process distribution, the probability of successful transmit is given by:

$\mathrm{P}_{\mathrm{S}}=\mathrm{e}^{-2 \mathrm{G}}$

where $\mathrm{L}=1$ (normalized)

The throughput (The expected number of successful transmission per unit time) is equal to:
$\mathrm{S}=\mathrm{Ge}^{-2 \mathrm{G}}$

It is obvious that the throughput can be improved if the vulnerable interval is minimized. For example, slotted Aloha reduces this period to $(\mathrm{L})$ yielding a throughput equal to $\left(\mathrm{Ge}^{-\mathrm{G}}\right)$. This 


\section{Jabbar : Applying Direct Sequence Spread Spectrum to Improve the Throughput of.......}

paper introduces spreading technique to further minimizing the vulnerable period and a throughput analogue to the CSMA protocol throughput for a certain range of offered load is expected.

\section{Spread Spectrum:}

Spread spectrum deals with different techniques which are applied to minimize the effect of jamming and/or noise effects. In this paper direct sequence technique is introduced. According to this technique, the data of different users are scrambled by different PN codes (each user has a unique PN code), then forwarded through a common channel[3].

At the receiver side, and in order to extract the data of a given user, the PN code of that user must be available. Figure (2) shown a simple correlator which is used to obtain the data of the intended user.

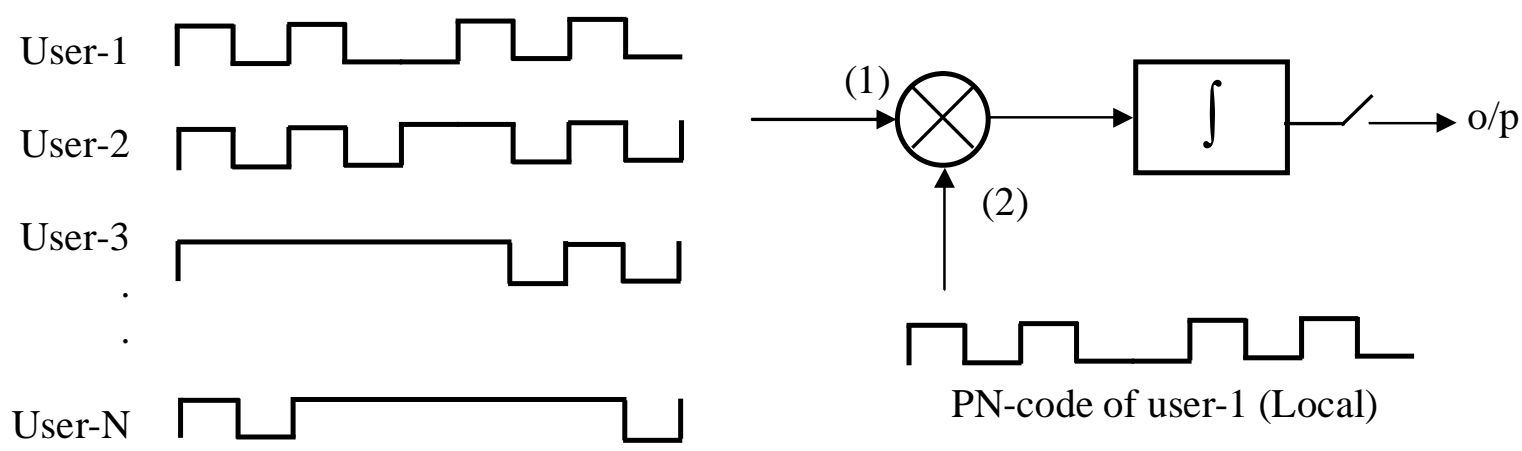

Figure (2): A simple correlator to extract the data of user-1

It is obvious that the scrambled data of user1 is correlated with the local PN code and high output is expected, while other users are cross-correlated with the PN code applied at point (2). In order to maximize the auto correlation and minimize the cross correlation. Al-Kasami PN code is chosen. It is characterized by a very low cross correlation[4]. Figure (3) shows the circuit which is used to generate this type of spreading code.
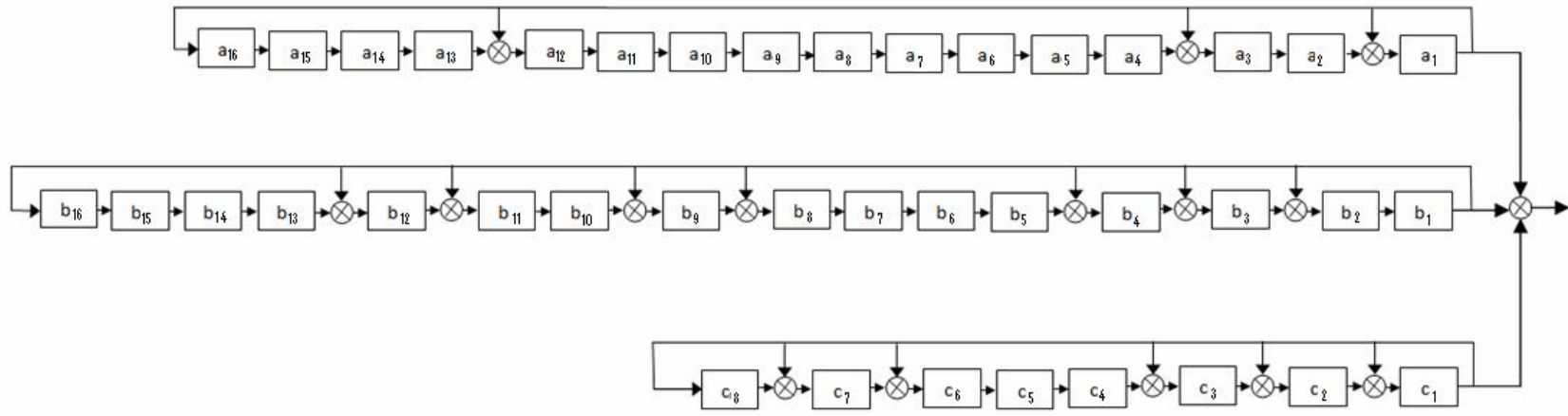

Figure(3). Al-Kasami PN code generator 


\section{$\begin{array}{lllll}\text { Al-Rafidain Engineering } & \text { Vol.17 } & \text { No.5 } & \text { October } 2009\end{array}$}

\section{Proposed Aloha protocols:}

A typical packet of data usually consists of header, payload and trailer, the header contains fields for destination, source and other important fields. protocol:

Two algorithms are suggested to improve the throughput performance of the Aloha

\section{a- Common PN code for all users:}

According to this algorithm, a ready packet must be applied to the input of the circuit shown in figure (4).
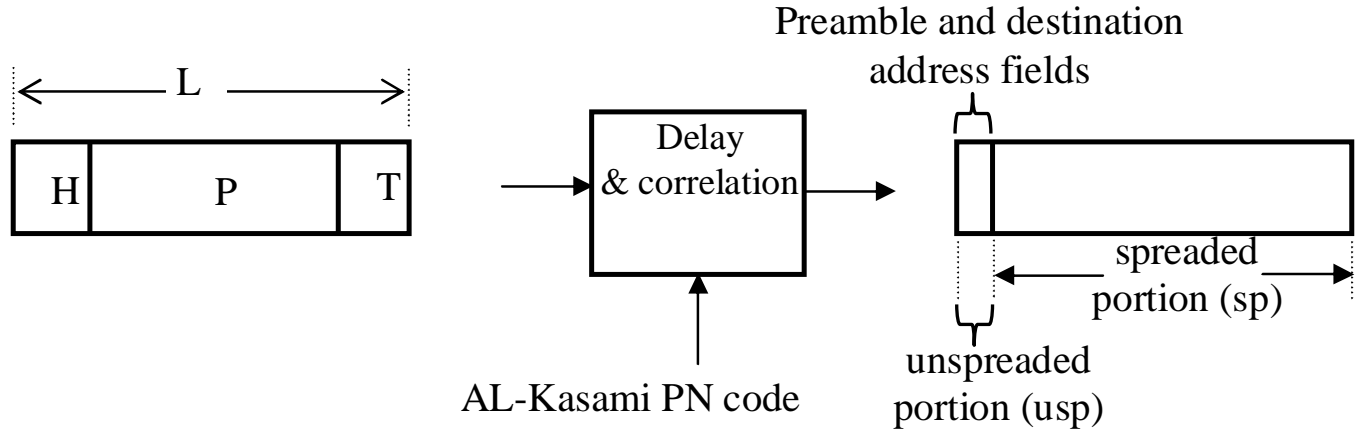

Figure (4) shows how to generate spreaded packet according to the common PN code algorithm.

The circuit shows that the output packet is spreaded except the preamble and destination address fields.

Since the PN code is common, then any transmitted packet must be received by all users. The user whose address placed in the destination field will accept the packet, while other users ignore it. The packet must be applied to a correlator circuit to demodulate (despread) the received packet. It is obvious that the vulnerable period (contention window) is now restricted to the unspreaded portion (usp) of the packet. So if no other users transmit their packets during the (usp), the packet is considered as a successful one.

On the other hand, if a packet arrived at the spreaded portion then it will have no effect due to the low cross correlation between the two packets, see figure (5).

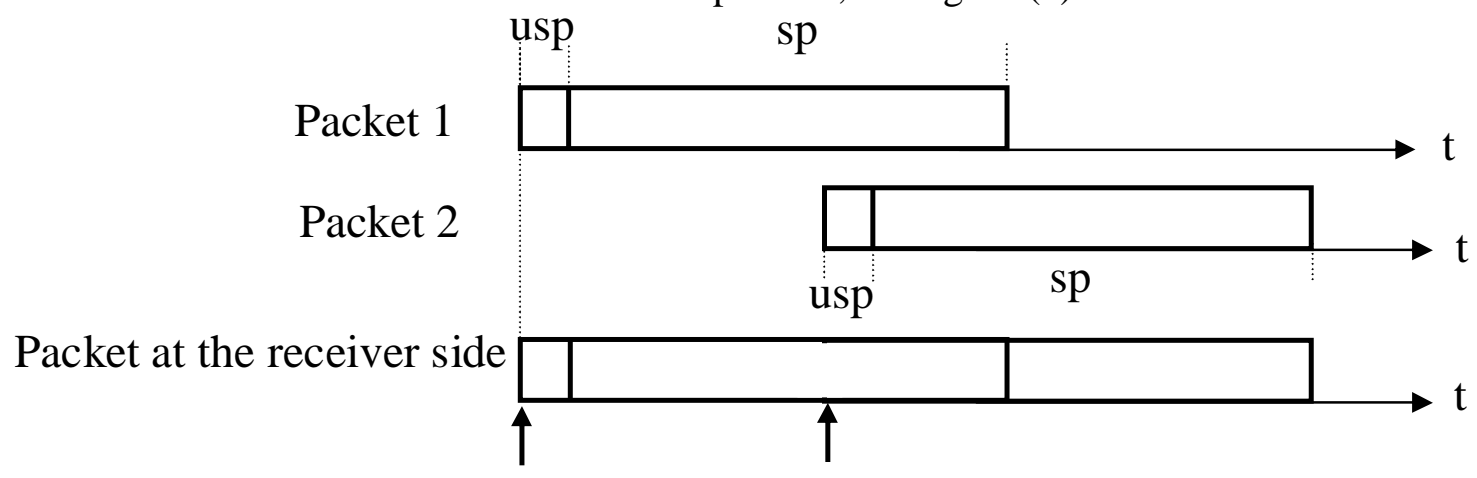

Figure (5) shows that the first packet is successful since there is no other packets arrived during the first usp

It is clear from figure (5) that the second packet is failed to be received successfully due to the interaction between the (usp) of the second packet with (sp) of the first one.

For simplicity of modeling, the time axis is divided into cycles, each one is divided into transmission period (TP) with variable length and a variable idle period (IP), see figure (6). 


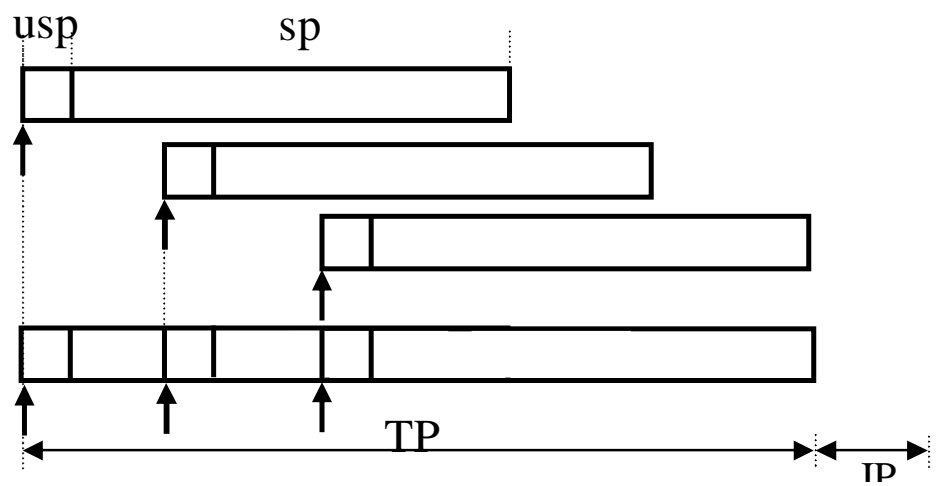

Figure (6) shows one possible successful cycle which consists of TP (multiple packets) and idle period (IP).

It is clear from Figure (6) that there is only one successful packet and two unsuccessful packets (or even more) during the cycle. The number of packets during a cycle depends on the offered load of the system $(G)$. It means that there is a high probability of one packet cycles at low offered load and the number of packets/cycle increases as the offered load increases.

Another possible packets distribution within a cycle is shown in figure (7). It is clear that there is no successful packet during the cycle because there is two packets started their transmission during the (usp) of the first packet.

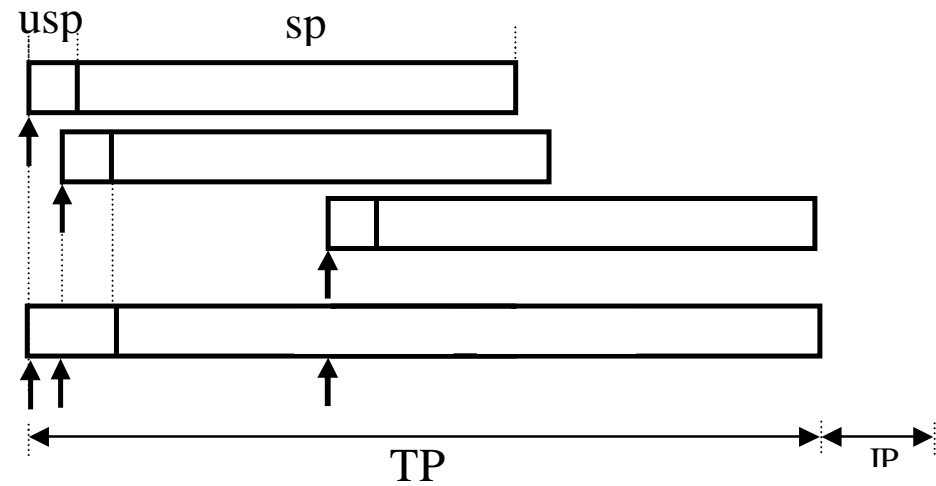

Figure (7) shows unsuccessful cycle (two packets arrived during usp)

From a statistical point of view, the probability of successful transmission of a packet is given by $\left(\mathrm{e}^{-\mathrm{tuG}}\right)$, where $(\mathrm{tu}=\mathrm{usp})$ and the packet length is normalized (i.e., $\left.\mathrm{L}=1\right)$. It is clear that the vulnerable period is now equal to (tu). Referring to Figure (8), the throughput of the proposed Aloha with common PN code can be derived as follows:

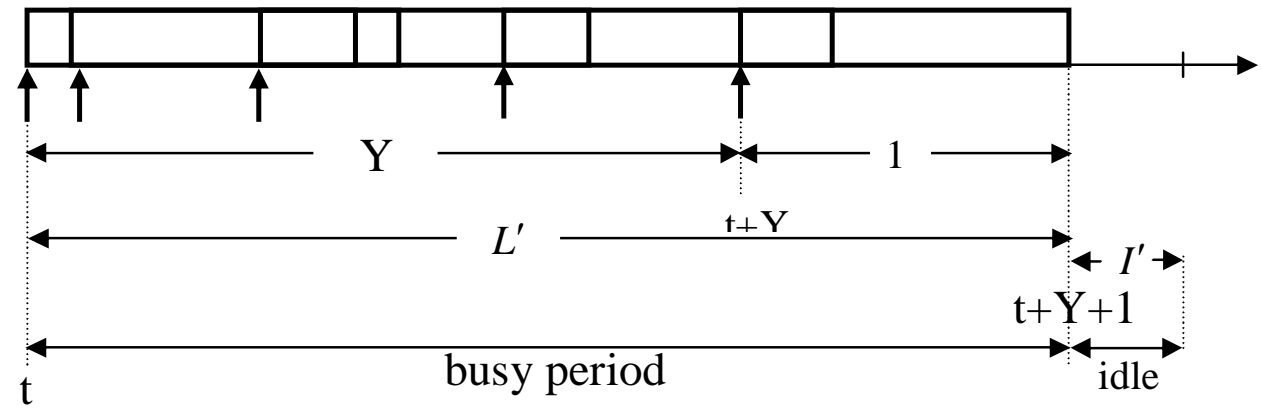

Figure (8) shows the details of a typical cycle. 


\section{$\begin{array}{llll}\text { Al-Rafidain Engineering } & \text { Vol.17 } & \text { No.5 } & \text { October } 2009\end{array}$}

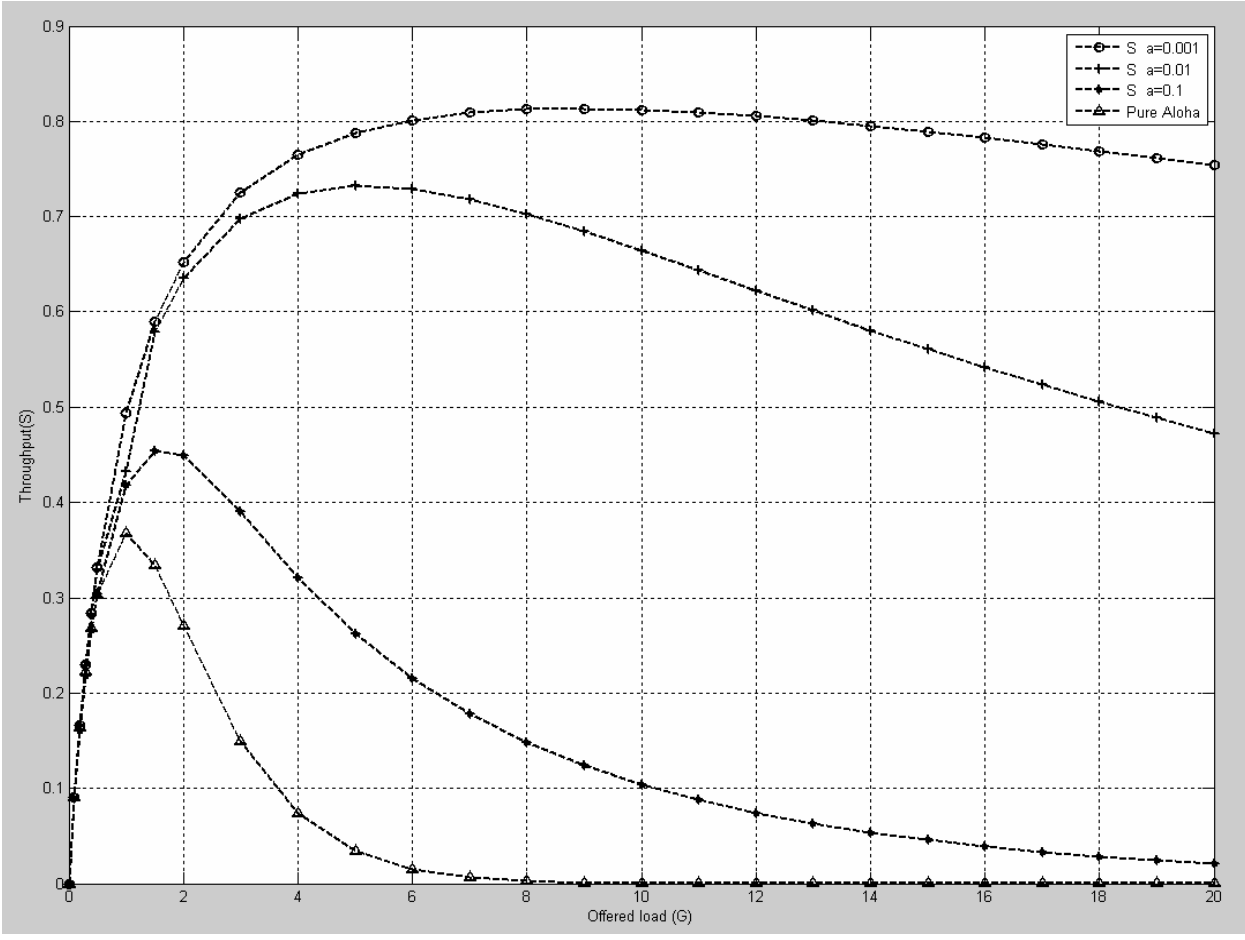

Figure (9) shows the throughput performance of the proposed Aloha with common PN code

Let $\mathrm{t}+\mathrm{Y}$ be the last packet arriving time between $\mathrm{t}, \mathrm{t}+\mathrm{Y}$. The transmission of all packets will be completed at $(\mathrm{t}+\mathrm{Y}+1)$. The last packet received will be delayed by (a) second (the propagation delay). Therefore, the transmission period is equal to $(\mathrm{Y}+1+\mathrm{a})$, during this period there is only one packet successful. The average duration of a busy period is $(1+\bar{Y}+\mathrm{a})$. The cumulative density function of $\mathrm{Y}$ is given by:

$P_{r}(Y \geq y)=P_{r}\left(\right.$ no arrival occurs in an int erval of width $\left.\left(L^{\prime}-y\right)\right)$

$$
\begin{aligned}
& =e^{-G\left(L^{\prime}-y\right)} \\
& =\int_{0}^{L^{\prime}} G y e^{-G\left(L^{\prime}-y\right)} d y \\
& =G e^{-G L^{\prime}} \int_{0}^{L^{\prime}} y e^{G y} d y \\
& =G e^{-G L^{\prime}}\left[\left.y \frac{e^{L^{\prime} G}}{G}\right|_{0} ^{L^{\prime}}-\int_{0}^{L^{\prime}} \frac{e^{G y}}{G} d y\right] \\
& =G e^{-G L^{\prime}}\left[\frac{L e^{L^{\prime} G}}{G}-\frac{e^{G L^{\prime}}-1}{G^{2}}\right] \\
& =L^{\prime}-\frac{1}{G}\left(1-e^{-G L^{\prime}}\right)
\end{aligned}
$$

Since the length of $L^{\prime}$ depends on $\mathrm{G}$, therefore, $L^{\prime}=\mathrm{a}(1+2 \mathrm{G})$ yields results consistent with the simulation result. 
Jabbar : Applying Direct Sequence Spread Spectrum to Improve the Throughput of.......

$\bar{Y}=a+2 a G-\frac{1}{G}\left(1-e^{-L^{\prime} G}\right)$

$\operatorname{Busy}\left(B^{\prime}\right)=1+\bar{Y}+a=1+2 a+2 a G-\frac{1}{G}\left(1-e^{-L^{\prime} G}\right)$

According to renewal theory, the throughput (S) is given by [1]:

$S=\frac{u^{\prime}}{B^{\prime}+I^{\prime}}$

$u^{\prime}=$ is the probability of no arrival during $(\mathrm{tu}+\mathrm{a})$

$I^{\prime}=$ The idle period which is exponentially distributed with an average value equal to $\left(\frac{1}{G}\right)$.

$S=\frac{e^{-(t u+a) G}}{1+2 a+2 a G-\frac{1}{G}\left(1-e^{-a(1+2 G) G}\right)+\frac{1}{G}}=\frac{e^{-(t u+a) G}}{1+2 a+2 a G+\frac{1}{G} e^{-a(1+2 G) G}}$

$S=\frac{G e^{-(t u+a) G}}{G(1+2 a+2 a G)+e^{-a(1+2 G) G}}$

Figure (9) shows the throughput performance of the proposed Aloha with common PN code for all users, the figure also includes the throughput performance of the conventional pure Aloha, the huge improvement of the performance is obvious.

\section{b- Multiple PN codes:}

This algorithm is more suitable to be implemented with slotted Aloha. According to the slotted Aloha, the time of the channel is divided into slots such that each slot can handle one packet.

A user can transmit his packet at the beginning of each slot only, this means that if the subscriber computer sense the channel to be busy then it must wait for the beginning of the next slot, this of course minimizes the possibility of collision as compared with the traditional Aloha (pure).

The possibly of collision arises if two or more subscribers transmit at the beginning of a given slot simultaneously, the maxim possible throughput of the slotted Aloha is equal to $(36.8 \%)[1]$.

According to the proposed multiple PN codes slotted Aloha, the system subscribers are divided into groups then assigning to each group, a PN code, this means that the collision domain is now divided by the number of PN codes to form smaller collision domains.

It is important to mention that the whole packet can be scrambled without losing the synchronization at the receiver side, (this is justified by the preserver of the channel time slots). Therefore, it is obvious that two subscribers with different PN codes can be existed at the same slot without contention due to the uncorrelated feature of the PN codes being used.

What is required for this algorithm to be elliceit is the preserver of a lockup table for the different PN codes, this will facilitate the communication between the different groups.

Figure (10) shows how two subscribers within two different groups can transfer packet between them. 
$\begin{array}{llll}\text { Al-Rafidain Engineering } & \text { Vol.17 } & \text { No.5 } & \text { October } 2009\end{array}$

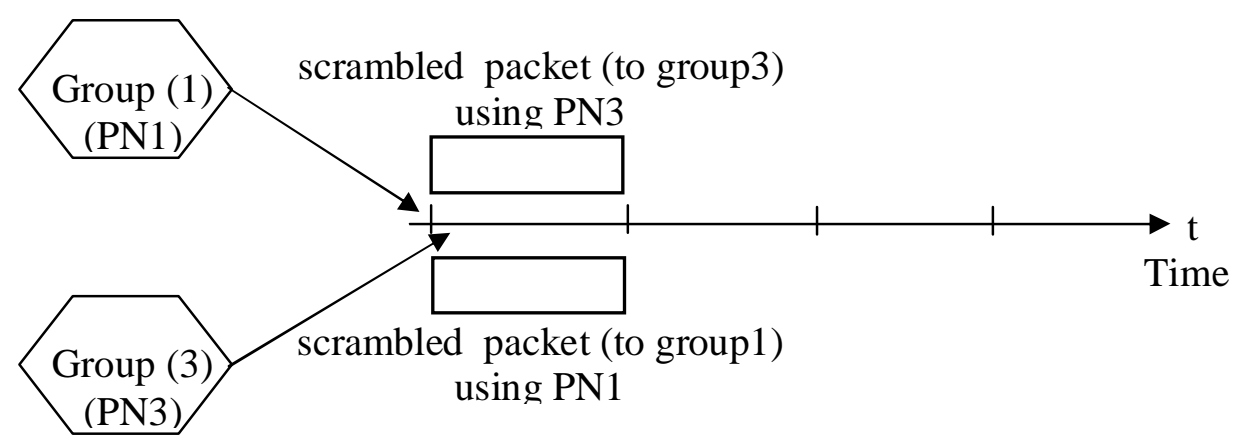

Figure (10) shows successful transmission of two packets within a given slot (the two packets one related to two different groups)

It is clear from Figure (10) that a user within group (1) uses the scrambling code of group (3) to transmit a packet during a communication session to a user within group (3), on the other hand a user within group (3) can send a packet to the user within group (1) using the scrambling code of group (1). Figure (10) also shows that the two users use the same slot without suffering from the collision problem (PN codes are uncorrelated).

From the mathematical point of view, conventional slotted Aloha vulnerable period is now restricted to the slot interval, this means that the probability of success (PS) is equal to $\left(\mathrm{e}^{-\mathrm{G}}\right)$, since the throughput $(\mathrm{S})$ is equal to $\mathrm{GP}_{\mathrm{S}}$, then:

$\mathrm{S}=\mathrm{Ge}^{-\mathrm{G}}$

The maximum value of $(\mathrm{S})$ is equal to $\left(\mathrm{e}^{-1}\right)$ or $0.368(36.8 \%)$ each group can reach this value of throughput. For example, if a main group is divided into five scrambled groups, then five subscribers from the different groups can transmit though the same slot. As mentioned before, a collision between users arise if two users within the same group try to transmit through a give slot using the same PN code (they belong to the same group). Accordingly, any scrambled group of the main group can reach a maximum throughput of $36.8 \%$, but it will never reach more than $7.36 \%$ if the scrambling of the groups is removed within the main group.

Fig. (11) shows the throughput of one of the groups with and without scrambling code within the main group.

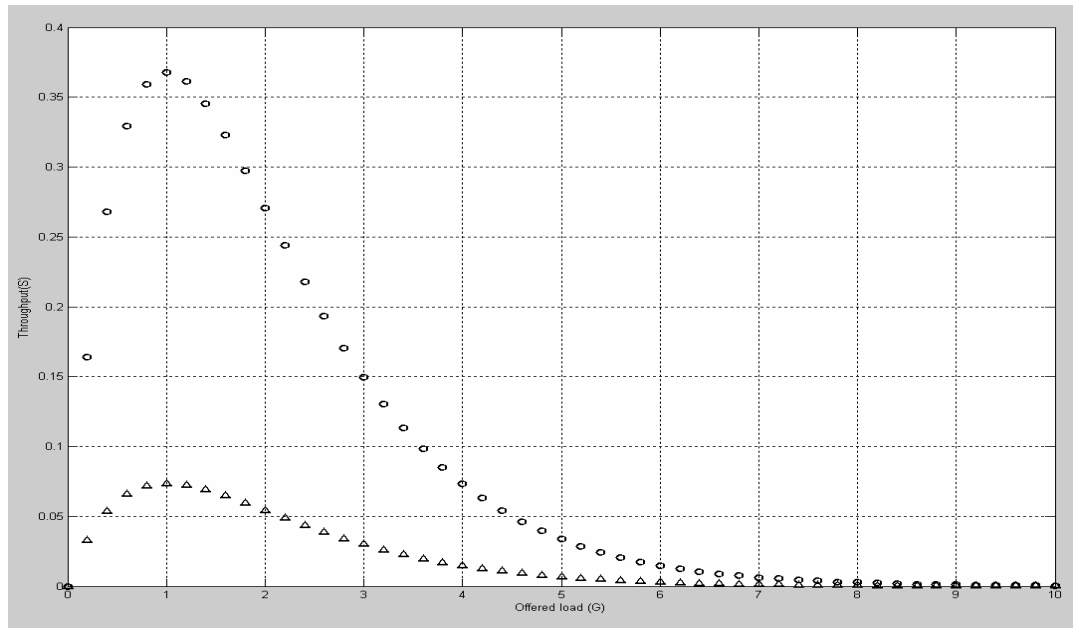

Figure (11) shows the throughput of the one of the groups within the main group with and without its scrambling code. 
Jabbar : Applying Direct Sequence Spread Spectrum to Improve the Throughput of.......

\section{Conclusion:}

The results of the proposed Aloha with common PN code shows that there is an excellent improvement in the throughput performance, but as the offered load increases, the effective length of the busy period will increase too yielding lower throughput performance. On the other hand, the throughput of the proposed Aloha with multiple PN code shows huge improvement of the throughput performance as the number of subgroups increases (i.e. minimizing the collision domain).

\section{Reference:}

[1] L. Kleinrock and F. A. Tobagi, " Packet switching in radio channels: part I- carrier sense multiple-Access modes and their throughput-Delay characteristics", IEEE Trans. Commun. Vol. COM-23, pp. 1400-1416, No-12 Dec. 1975.

[2] V. O. K. Li, " Multiple Access Communication Network", IEEE Commun. Magazine, Vol. 25, No. 6, pp. 41-48, June 1987.

[3] P. Z. and Borth, " Introduction to spread spectrum communication", Prentice-Hall, 1995.

[4] Esmael H. Dinan and Bijan Jabbari, " Spreading codes for direct sequence CDMA and wideband CDMA cellular networks", George Mason university, 1998. 